\title{
Effect of the combination of astragaloside IV and Panax notoginseng saponins on pyroptosis and necroptosis in rat models of cerebral ischemia-reperfusion
}

\author{
BIAO TANG ${ }^{*}, \mathrm{XU} \mathrm{SHE}^{*}$ and CHANG-QING DENG \\ Department of Physiology, Medical School, Hunan University of Chinese Medicine, \\ Changsha, Hunan 410208, P.R. China
}

Received November 20, 2020; Accepted June 28, 2021

DOI: $10.3892 /$ etm.2021.10557

\begin{abstract}
Pyroptosis and necroptosis are closely associated with the mechanism underlying cerebral ischemia-reperfusion (I/R) injury. The combination of astragaloside IV (AST IV) and Panax notoginseng saponins (PNS) has remarkable effects on the alleviation of cerebral I/R damage. However, whether inhibition of pyroptosis and necroptosis is the mechanism underlying the beneficial effects of this drug combination on cerebral I/R injury remains unclear. To explore the effects and mechanisms of drug treatment, middle cerebral artery occlusion was performed to induce I/R injury in rats, which was verified based on neurological deficit score (NDS), infarct volume and H\&E staining. Activation of pyroptosis and necroptosis was detected by western blot analysis of associated proteins. The results of the present study demonstrated that treatment with AST IV and PNS, either alone or in combination, significantly reduced the NDS, cerebral infarct volume and cell injury rate in the cerebral cortex of rats. The treatments also improved pathological injury to the cerebral cortex and reduced the levels of proteins associated with pyroptosis and necroptosis. These effects were stronger in the combination drug group compared with groups treated with a single drug alone. The findings of the present study suggested that the combination of AST IV and PNS exhibited stronger neuroprotective effects in I/R injury than either drug alone, and that the underlying mechanism was associated with inhibition of pyroptosis and necroptosis.
\end{abstract}

Correspondence to: Professor Chang-Qing Deng, Department of Physiology, Medical School, Hunan University of Chinese Medicine, 300 Xueshi Road, Changsha, Hunan 410208, P.R. China

E-mail: dchangq@sohu.com

*Contributed equally

Key words: astragaloside-IV, Panax notoginseng saponins, combination, pyroptosis, necroptosis, cerebral ischemia-reperfusion

\section{Introduction}

Cerebral ischemia-reperfusion $(\mathrm{I} / \mathrm{R})$ is a common feature of ischemic stroke, and its pathogenesis is associated with oxidative stress, apoptosis and the inflammatory response (1). A previous report demonstrated that pyroptosis and necroptosis are commonly associated with the inflammatory response (2-5). Notably, both pyroptosis and necroptosis aggravate damage during cerebral I/R and are implicated in the regulation of inflammation. Gasdermin D (GSDMD), a molecule involved in the execution of pyroptosis, is activated by the nucleotide-binding oligomerization domain, leucine-rich repeat and pyrin domain containing 3 (NLRP3) inflammasome. GSDMD is then cleaved, producing the GSDMD N-terminal (GSDMD-N) fragment, which forms polymers and channels on the cell membrane. Necroptosis activation begins with the recruitment of receptor-interacting protein kinase (RIP)1 and RIP3 (2,3). Subsequently, RIP3 phosphorylates mixed lineage kinase domain-like protein (MLKL), and the resulting phosphorylated (p)-MLKL protein oligomerizes on the cell membrane and forms channels $(2,3)$. Consequently, both pyroptosis and necroptosis lead to the emission of pro-inflammatory factors or cell rupture through these channels, which causes an inflammatory response $(4,5)$ and cerebral I/R injury (6-8). Pyroptosis and necroptosis are reported to induce cerebral I/R, and the inhibition of either one reduces cerebral I/R injury. This indicates that the suppression of pyroptosis and necroptosis has the potential to act as an important target for cerebral I/R injury intervention (6-8).

In Traditional Chinese Medicine (TCM), it is believed that the combination of the herbal TCMs Astragalus membranaceus and Panax pseudoginseng nourish Qi and activate blood circulation, and the combination is used to treat cardiovascular and cerebrovascular diseases. This combination has been demonstrated as clinically effective in the treatment of stroke (9). The main components of A. membranaceus and P.pseudoginseng are astragaloside IV (AST IV) and $P$. notoginseng saponins (PNS), respectively. These components strongly suppress inflammation in cerebrally ischemic rats and alleviate cerebral I/R injury (9-12). However, whether AST IV and PNS effectively suppress pyroptosis and necroptosis remains to be elucidated. In the current study, AST IV and PNS were used 
to treat rats with middle cerebral artery occlusion (MCAO) by neurological evaluation, 2,3,5-triphenyltetrazolium chloride (TTC) and hematoxylin and eosin (H\&E) staining and western blot analysis to investigate the neuroprotective effects of these drugs and to elucidate their underlying mechanisms.

\section{Materials and methods}

Animals. A total of 80 healthy male specific-pathogen-free (SPF) Sprague-Dawley rats (age, 6-7 weeks; weight, 230-250 g) were provided by Hunan Slake Jingda Experimental Animal Co., Ltd. (production license no. 2017-0004). Animals were housed under SPF conditions at the Experimental Animal Center of Hunan University of Chinese Medicine on a 12-h light/dark cycle, at an ambient temperature of $25 \pm 1^{\circ} \mathrm{C}$ and $60 \%$ humidity. After 1 week of adaptive feeding ( $12.5 \mathrm{~g}$ food/12 h), rats were made to fast with free access to water for $12 \mathrm{~h}$ prior to the experiments.

Chemicals. AST IV (molecular formula, $\mathrm{C}_{41} \mathrm{H}_{68} \mathrm{O}_{14}$; molecular weight, 784; purity, $\geq 98.05 \%$; cat. no. MUST-14102910), Notoginsenoside $\mathrm{R}_{1}$ (cat. no. MUST-A0760), Ginsenoside $\operatorname{Rg}_{1}$ (cat. no. MUST-A0237), Ginsenoside Re (cat. no. MUST-A0244), Ginsenoside $\mathrm{Rb}_{1}$ (cat. no. MUST-A0234), Ginsenoside Rd (cat. no. MUST-A0245) andPNS (cat. no. DST200706-054) were purchased from Chengdu Must Biotechnology Co., Ltd. TTC (cat. no. BCBP3272V) was purchased from Sigma-Aldrich (Merck KGaA). Western blotting buffer (cat. no. P0013) were purchased from Shanghai Biyuntian Biotechnology Co., Ltd. Protease inhibitor cocktail (without EDTA; 100X DMSO; cat. no B14002) and phosphate inhibitor cocktail (100X; cat. no. B15002) were purchased from Bimake.com, and rabbit polyclonal anti-IL-1 $\beta$ (cat. no. ab9787) and anti-IL-18 (cat. no. ab191860) were purchased from Abcam. Rabbit polyclonal anti-caspase 1 (cat. no. NBP1-45433) and anti-NLRP3 (cat. no. NBP2-12446) were purchased from Novus Biologicals. Rabbit monoclonal anti- $\beta$-actin (cat. no. A2228) was purchased from Sigma-Aldrich (Merck KGaA), and goat anti-rabbit (cat. no. AP132P) and goat anti-mouse (cat. no. AP124P) secondary antibodies were purchased from Merck KGaA. Embolism thread (cat. no. 2636A2; head diameter, $0.36 \pm 0.02 \mathrm{~mm}$ ) was purchased from Beijing Xilong Technology Co., Ltd.

High-performance liquid chromatography (HPLC). For quantitative determination, standard stock solutions of five pure saponins, including Notoginsenoside $\mathrm{R}_{1}$, Ginsenoside $\mathrm{Rg}_{1}$, Ginsenoside $\mathrm{Re}$, Ginsenoside $\mathrm{Rb}_{1}$ and Ginsenoside $\mathrm{Rd}$, were individually prepared in methanol. The solutions were then mixed and diluted to different concentrations for the standard curves. The air-dried PNS was powdered in a mill and then immersed in $600 \mathrm{ml}$ distilled water at $50^{\circ} \mathrm{C}$ for $30 \mathrm{~min}$ in a water bath. Following the addition of $4,000 \mathrm{ml}$ absolute ethanol, the PNS was ultrasonically extracted twice for $1 \mathrm{~h}$ each. The temperature of the ultrasonic bath was maintained $\left(25 \pm 2^{\circ} \mathrm{C}\right)$ with running water. Following filtration, the combined ethanol extracts were concentrated to dryness under reduced pressure in a rotary evaporator at $55^{\circ} \mathrm{C}$. The residue was reconstructed in $100 \mathrm{ml}$ distilled water. HPLC analysis was performed on a Waters Alliance e2695 HPLC system (Waters Corporation). The chromatographic separation was performed on a reversed-phase Zorbax $\mathrm{C} 8$ column at a temperature of $35^{\circ} \mathrm{C}$. The mobile phase consisted of acetonitrile (solvent A) and water (solvent B), eluted with a gradient program as follows: 0-5 min, 15-30\% A; 5-15 min, 30-32\% A; 15-35 min, 32-32\% A; 35-45 min, 32-38\% $\mathrm{A}$ and $45-60 \mathrm{~min}, 38-50 \% \mathrm{~A}$. The flow rate was maintained at $0.8 \mathrm{ml} / \mathrm{min}$. The detection wavelength was set at $203 \mathrm{~nm}$. The five primary saponins were completely separated within $45 \mathrm{~min}$ without obvious interference. The retention times of Notoginsenoside $\mathrm{R}_{1}$, Ginsenoside $\mathrm{Rg}_{1}$, Ginsenoside $\mathrm{Re}$, Ginsenoside $\mathrm{Rb}_{1}$ and Ginsenoside $\mathrm{Rd}$ were 16.32, 23.45, 24.53, 36.57 and $40.13 \mathrm{~min}$, respectively. The molecular structure, compound identification, molecular formula and weight of the five primary saponins were obtained from PubChem (pubchem. ncbi.nlm.nih.gov/).

Experimental groups and procedures. Rats were randomly divided into five groups: i) Sham group, ii) model group, iii) AST IV group, iv) PNS group and v) combination group. A total of 120 rats were used, 8 of which died due to unplanned hemorrhage during surgery; 7 died due to intracranial hemorrhage and brain injury after surgery, and the remaining rats survived until being euthanized. Each group comprised 21 rats ( 8 for brain H\&E staining, 8 for TTC staining and 5 for western blot analysis). The dosage and method of administration for AST IV $(28 \mathrm{mg} / \mathrm{kg})$ and PNS $(80 \mathrm{mg} / \mathrm{kg})$ alone or in combination were based on previous studies $(10,11,13)$. Drugs were administered three times $(10 \mathrm{ml} / \mathrm{kg}$ per time) by gavage in all groups. Optimal administration times were 36 and $12 \mathrm{~h}$ before model establishment and $12 \mathrm{~h}$ after model establishment, and the sham and model groups received equal volumes of physiological saline. Surgery was performed after anesthetizing rats with an intraperitoneal (i.p.) injection of $10 \%$ chloral hydrate $(300 \mathrm{mg} / \mathrm{kg}$; cat. no. 8MQ20-CC; Tixiai Huacheng Industrial Development Co., Ltd.). Rats exhibited no sign of peritonitis post-injection. A total of 105 rats remained alive $24 \mathrm{~h}$ after surgery. Following this, the rats were euthanized by i.p. injection of sodium pentobarbital (140 mg/kg body weight). Death was monitored based on cardiac activity and respiration. All efforts were taken to minimize animal suffering and reduce the number of animals used.

MCAO model establishment. The MCAO model was established as previously described (13). Following anesthetization, the right common carotid artery (CCA), external carotid artery (ECA) and internal carotid artery (ICA) were isolated through a neck incision. Surgical thread was used to ligate the distal portion of the ECA, and ECA and its branches were disconnected near the ligation site using electrocoagulation. A slipknot was made to ligate the distal part of the ICA, and an arterial clamp was used to clamp the distal ICA and CCA. A small orifice was made in the ECA stump, and an embolism thread was introduced into the ECA and directed into the ICA through the bifurcation of the CCA. The artery clip that had been used to clamp the distal ICA was removed, and the embolism thread was inserted into the intracranial portion of the ICA at a distance of $18 \pm 2 \mathrm{~mm}$ from the distal portion of the CCA bifurcation. When a slight resistance to insertion of the thread into the anterior cerebral artery was felt, insertion was stopped. Finally, the ICA was clamped to prevent bleeding and movement, and the neck skin was sutured. Following $2 \mathrm{~h}$ of blood flow blocking and a subsequent $24 \mathrm{~h}$ of reperfusion, follow-up experiments were conducted. In the sham group, the CCA, ECA and ICA were isolated without insertion of an embolism thread. 
Neurological deficit score (NDS) test. Following $22 \mathrm{~h}$ reperfusion, the NDS of each rat in each group was calculated, as previously described (13). Briefly, the characterization procedure was as follows: 0, asymptomatic or no neurological deficit; 1 , when lifting the tail, the rats' left forelimb could not be extended; 2 , when walking, rats are circling to the contralateral side; 3 , when walking, rats are tilting to the contralateral side; and 4, no spontaneous motor activity or loss of consciousness.

TTC staining. Following $24 \mathrm{~h}$ of reperfusion, 8 rats/group were anesthetized and subsequently euthanized. The whole brain was rapidly removed from each rat, harvested and placed in a $-20^{\circ} \mathrm{C}$ freezer for $20 \mathrm{~min}$. From the beginning of the optic chiasm, the coronal plane was sectioned at intervals of $2 \mathrm{~mm}$, which yielded exactly five slices. The slices were incubated in $2 \%$ TTC phosphate buffer and immersed in a constant-temperature water bath in the dark at $37^{\circ} \mathrm{C}$ for $15 \mathrm{~min}$, with stirring performed every $5 \mathrm{~min}$. After staining, brain slices were fixed with $4 \%$ paraformaldehyde at $4^{\circ} \mathrm{C}$ for $24 \mathrm{~h}$. Non-ischemic regions appeared red, whereas infarcted regions were pale. ImagePro Plus software version 6.0 (Media Cybernetics, Inc.) was used to calculate the non-ischemic red-stained TTC areas of the cerebral hemispheres on the normal and ischemic sides of each slice of brain. The corrected ischemic area value was obtained by subtracting the two areas in accordance with a previously described method (14) to eliminate the effect of cerebral edema on the infarction volume. The area of each ischemic cerebral hemisphere was multiplied by its thickness $(2 \mathrm{~mm})$ and this value was added to find the entire approximate cerebral infarction volume. Finally, this approximate value was divided by the normal hemisphere volume to calculate the infarction volume rate. As previously described (14), the following formula was used to calculate cerebral infarction volume rate: (Total contralateral hemisphere area-ipsilateral hemisphere area)/(total contralateral hemisphere area).

$H \& E$ staining. Following $24 \mathrm{~h}$ of reperfusion, 40 rats were anesthetized and euthanized. Whole brains were fixed in $4 \%$ paraformaldehyde overnight at room temperature and sliced into 2-mm coronal sections on the optic cross-plane. The brains were subsequently sliced to $3 \mu \mathrm{m}$ thickness, dewaxed with xylene and hydrated using a series of graded concentrations of ethanol (100, $5 \mathrm{~min}$; 95, $1 \mathrm{~min}$; 80, $5 \mathrm{~min}$; $75 \%$ ethanol, $5 \mathrm{~min}$; distilled water, $2 \mathrm{~min}$ ). Following washing three times with distilled water for 5 min each, sections were stained with hematoxylin for $10 \mathrm{~min}$, washed with running water for $10 \mathrm{~min}$ and counterstained with eosin for $5 \mathrm{~min}$ at room temperature. All sections were fixed on polylysine-treated glass slides for H\&E staining. The stained sections were observed under a light microscope (magnification, $\mathrm{x} 400$ ), and the cell damage ratio was calculated; five non-overlapping visual fields were randomly selected for observation of damaged cells in the ischemic cortex, and the total count of quantified cells was 300. Cell vacuolar degeneration, eosinophilic degeneration, nuclear contraction and nuclear dissolution were the main characteristics of damaged cells. The damaged cells and total cells in each high-magnification field were counted and the cell damage rate was calculated as follows, to obtain a percentage: (number of damaged cells/total number of cells) x100.
Western blot analysis. Western blotting was conducted to detect NLRP3-induced activation of pyroptosis and necroptosis. The cerebral ischemic penumbras of 5 rats from each group were weighed and homogenized, and $100 \mathrm{mg}$ of cerebral cortex from the ischemic penumbra was added to $10 \mathrm{X}$ pyrolysis liquid with a phosphatase inhibitor and protease inhibitor cocktail. The lysate was centrifuged at $12,000 \mathrm{x}$ g for $10 \mathrm{~min}$ at $4^{\circ} \mathrm{C}$, the pellet was discarded and the protein concentration in the supernatant was determined using a BCA assay. A total of $40 \mu \mathrm{g}$ protein/lane were separated by $8-15 \%$ SDS-PAGE and transferred to PVDF membranes (cat. no. ISEQ00010; Merck KGaA). The membranes were blocked in 5\% non-fat milk in TBS-Tween-20 buffer (100 mM NaCl; 10 mM Tris-HCl; pH, 7.4; 0.1\% Tween-20) for $2 \mathrm{~h}$ at room temperature and subsequently incubated overnight at $4^{\circ} \mathrm{C}$ with anti- apoptosis-associated speck-like protein containing a caspase activation and recruitment domain (ASC; 1:500; cat. no. NBP1-45453; Novus Biologicals), anti-NLRP3 (1:1,000), anti-IL-1 $\beta$ (1:1,000), anti-IL-18 (1:1,000), anti-caspase-1 (1:500), anti-RIP1 (1:1,000; cat.no.ab106393; Abcam), anti-RIP3 (1:1,000; cat. no. ab56164; Abcam), anti-MLKL (1:1,000), anti-p-MLKL $(1: 1,000)$, anti-GSDMD (1:1,000), anti-GSDMD-N $(1: 1,000)$ and anti- $\beta$-actin $(1: 5,000)$. Following a wash with PBS, the membranes were incubated with horseradish peroxidase-labeled secondary antibody $(1: 10,000)$ for $1 \mathrm{~h}$ at room temperature. Protein bands were detected using an enhanced chemiluminescence detection kit (cat. no. 631701; Pierce; Thermo Fisher Scientific, Inc.), and the integrated optical density (IOD) of the target protein was determined using Quantity One 1-D analysis software (Bio-Rad Laboratories, Inc.; version 4.6.8). $\beta$-actin was used as the internal reference and the ratio of the target protein IOD to the $\beta$-actin IOD was calculated.

Statistical analysis. The data were analyzed using SPSS software version 22.0 (IBM Corp.). All continuous data are expressed as the mean \pm standard deviation. Differences among the experimental groups were evaluated using one-way ANOVA followed by Tukey's post hoc test to compare the differences between groups. For NDS data, values are presented as the median (interquartile range), and groups were analyzed using Kruskal-Wallis test followed by Dunn's multiple comparisons test. $\mathrm{P}<0.05$ was considered to indicate a statistically significant difference.

\section{Results}

PNS component identification. HPLC was used to identify PNS compounds by measuring the absorbance at $203 \mathrm{~nm}$. The main saponins of PNS were completely separated without interference. The major effective constituents included: i) $6.96 \%(\mathrm{v} / \mathrm{v})$ notoginsenoside $\mathrm{R}_{1}$; ii) $34.29 \%$ (v/v) ginsenoside $\mathrm{Rg}_{1}$; iii) $2.65 \%$ (v/v) ginsenoside Re; iv) $41.34 \%$ (v/v) ginsenoside $\mathrm{Rb}_{1}$; and v) $10.19 \%(\mathrm{v} / \mathrm{v})$ ginsenoside $\mathrm{Rd}$. The chemical fingerprint and molecular structures of PNS are displayed in Fig. 1.

AST IV and PNS reduce cerebral infarction volume and NDS in rats with cerebral I/R. TTC staining of brain tissue revealed no infarct areas nor clear neurological deficits in sham group rats (Fig. 2A). However, rats in the MCAO model group presented large areas of pale infarction as well as neurological deficits, with larger cerebral infarction volumes and higher NDSs 


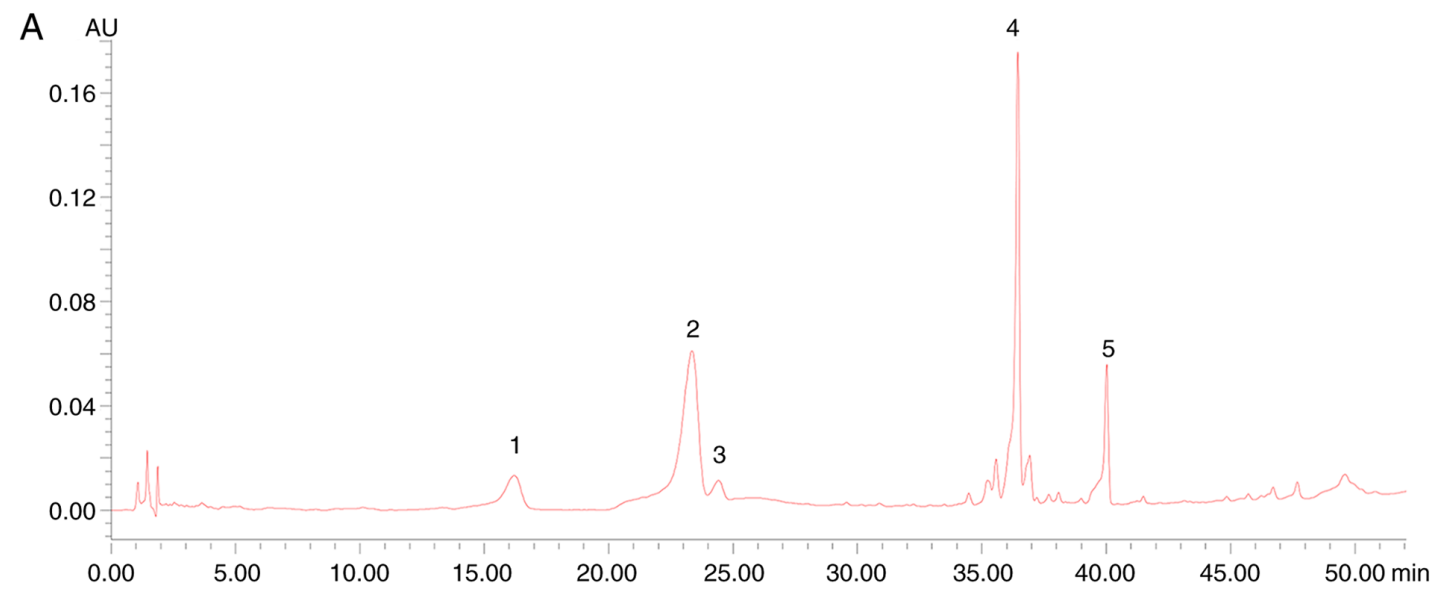

B

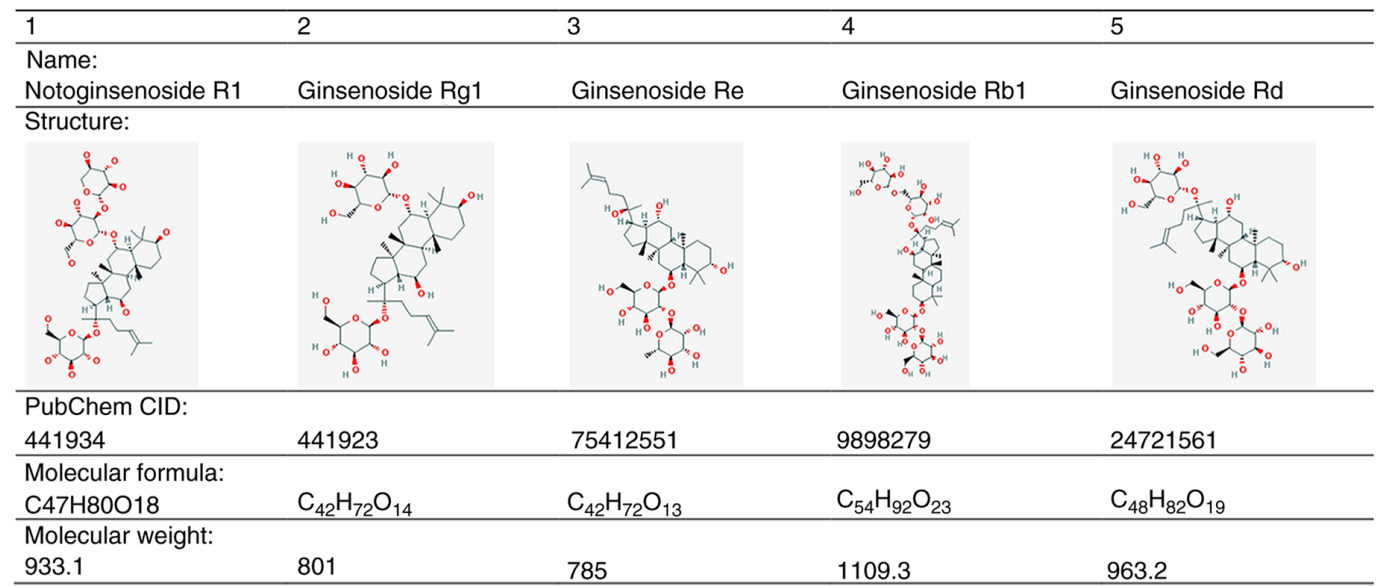

Figure 1. PNS composition identification. (A) PNS components are indicated as follows: 1 , Notoginsenoside $\mathrm{R}_{1}$; 2, ginsenoside $\mathrm{Rg}_{1}$; 3 , ginsenoside Re; 4, ginsenoside $\mathrm{Rb}_{1}$; and 5, ginsenoside Rd. (B) PNS component identification detailing the molecular structure, PubChem CID, molecular formula and weight. CID, compound identification; PNS, Panax notoginseng saponins.

A

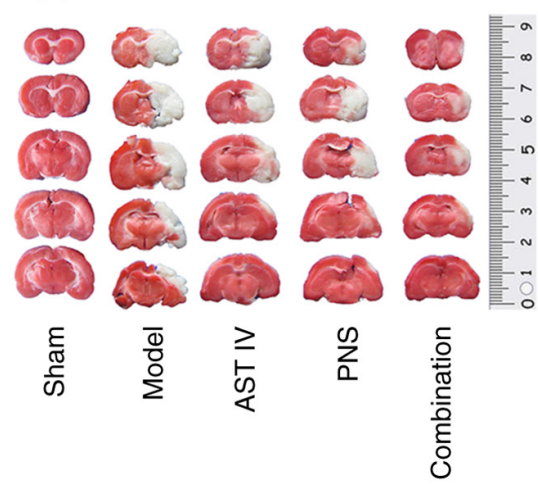

B

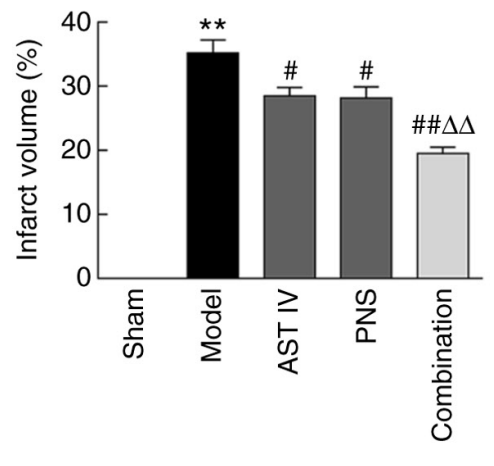

C

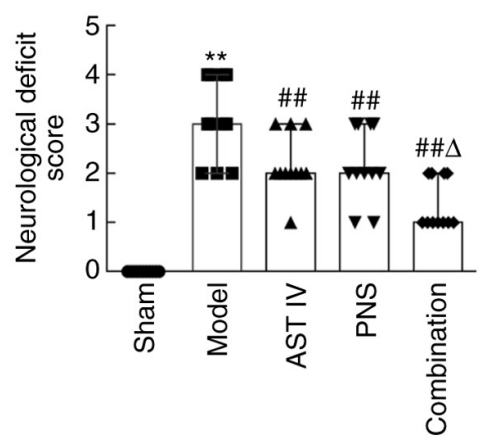

Figure 2. AST IV and PNS decrease cerebral infarction volume and the NDSs in rats with cerebral ischemia-reperfusion. (A) TTC-stained brain slices; pale areas indicate infarction $(\mathrm{n}=8)$. (B) Cerebral infarct volumes were calculated using TTC staining of brain sections ( $\mathrm{n}=8 \mathrm{rats} / \mathrm{group})$. (C) Functional deficits were examined via NDS ( $n=11$ rats/group). The groups are as follows: i) Sham, rats with sham operation; ii) model, rats with MCAO; iii) AST IV, rats with $\mathrm{MCAO}+28 \mathrm{mg} / \mathrm{kg}$ AST IV; iv) PNS, rats with MCAO + $80 \mathrm{mg} / \mathrm{kg} \mathrm{PNS}$; and v) combination, rats with $\mathrm{MCAO}+28 \mathrm{mg} / \mathrm{kg}$ AST IV + $80 \mathrm{mg} / \mathrm{kg} \mathrm{PNS}$. ${ }^{* *} \mathrm{P}<0.01$ vs. Sham; ${ }^{\#} \mathrm{P}<0.05,{ }^{\# \#} \mathrm{P}<0.01$ vs. Model group; ${ }^{\Delta} \mathrm{P}<0.05,{ }^{\Delta} \mathrm{P}<0.01$ vs. AST IV or PNS. AST IV, astragaloside IV; MCAO, middle cerebral artery occlusion; TTC, 2,3,5-triphenyltetrazolium chloride; NDS, neurological deficit score; PNS, Panax notoginseng saponins.

compared with those of rats in the sham group (Fig. 2A-C). Further results indicated that treatment with AST IV and PNS alone or in combination significantly decreased both the volume of cerebral infarction (Fig. 2A and B) and the relative
NDS (Fig. 2C) compared with the model group. Moreover, the volume of cerebral infarction and the NDSs were decreased significantly in the combination group compared with the AST IV and PNS groups alone (Fig. 2A-C). 

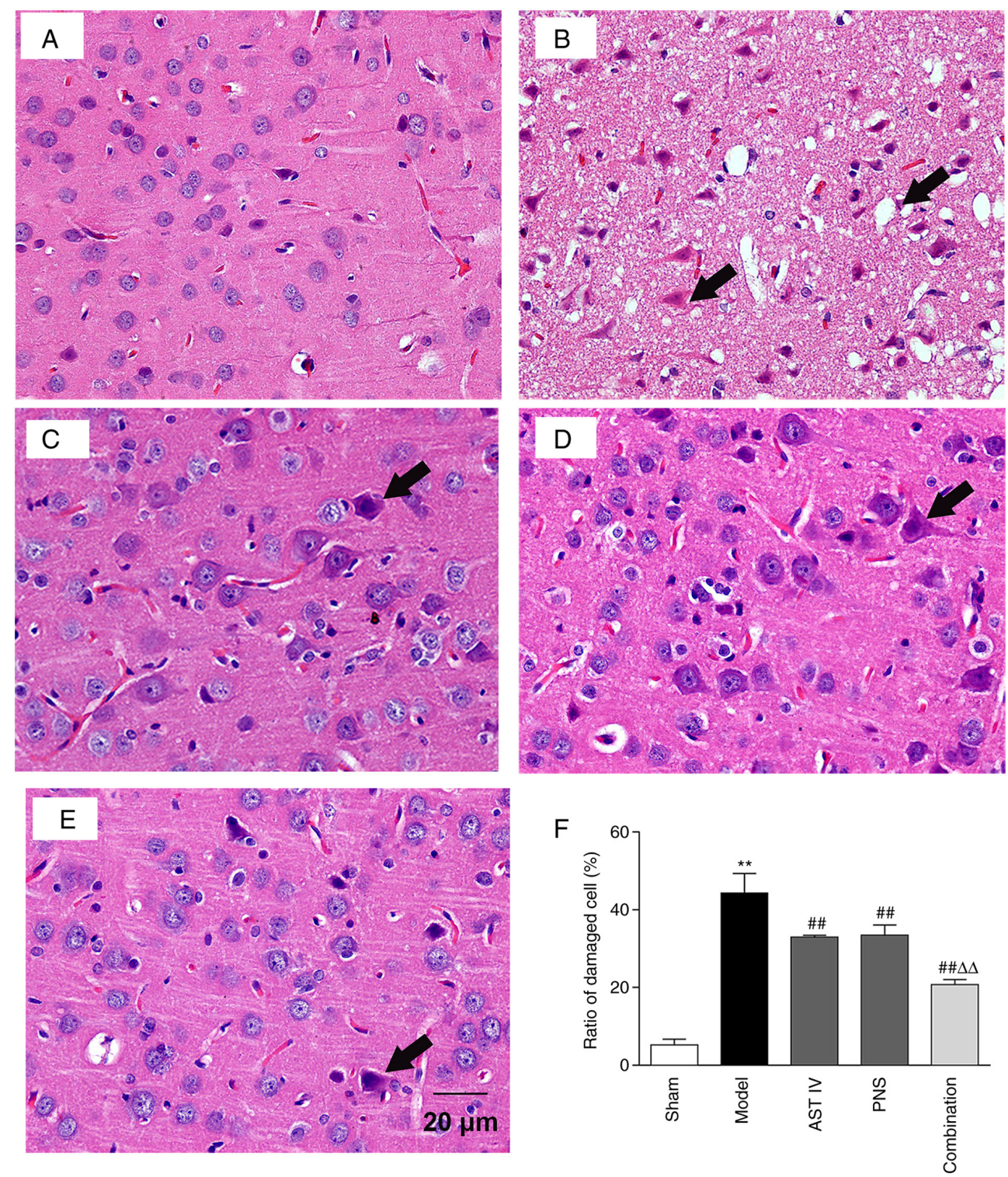

Figure 3. Combination treatment with AST IV and PNS alleviates cerebral cortical cell injury in rats. H\&E staining analysis of pathological changes in the cerebral cortices of rats in the. Black arrows indicate damaged cells. (A) sham, (B) model, (C) AST IV, (D) PNS and (E) combination groups (n=8 rats/group). (F) Damaged cell ratio. The groups are as follows: i) Sham, rats with sham operation; ii) model, rats with MCAO; iii) AST IV, rats with MCAO + 28 mg/kg AST IV; iv) PNS, rats with MCAO + $80 \mathrm{mg} / \mathrm{kg}$ PNS; and v) combination, rats with MCAO + $28 \mathrm{mg} / \mathrm{kg}$ AST IV + $80 \mathrm{mg} / \mathrm{kg}$ PNS. ${ }^{* *} \mathrm{P}<0.01$ vs. Sham; ${ }^{\# \#} \mathrm{P}<0.01 \mathrm{vs}$. Model; ${ }^{\Delta \Delta} \mathrm{P}<0.01$ vs. AST IV or PNS. AST IV, astragaloside IV; MCAO, middle cerebral artery occlusion; PNS, Panax notoginseng saponins.

Combination of AST IV and PNS reduces cerebral cortical-cell injury in rats. H\&E-stained brain tissue slices were observed under a light microscope. The results demonstrated that the cellular structure of the cerebral cortex in the sham group was complete. That is, nucleoli were clearly visible with regular structures and there was no interstitial inflammatory-cell infiltration; instead, there was a tight cell arrangement and occasional injury (Fig. 3A). However, following MCAO treatment, the pericellular space in the cerebral cortex was widened; the nuclei were irregular, and capillary atrophy, clear interstitial edema and a disordered cell arrangement were observed. An example of the damaged cells along with their site of vacuolation are displayed in Fig. 3B. Moreover, nuclei were hyperchromatic and condensed or displayed acidophilic degeneration. The results further demonstrated that the ratio of damaged cells in the cerebral cortex was significantly higher in the model group compared with the sham group (Fig. 3F).
Compared with the model group, cells in the AST IV, PNS and combination groups were mildly swollen, and the widened pericellular space had narrowed (Fig. 3C-E, respectively). In addition, acidophilic degeneration of nuclei was reduced, and cell damage was attenuated compared with the model group (Fig. 3C-F). Furthermore, cell damage was significantly decreased in the combination group compared with the AST IV and PNS groups (Fig. 3F).

Combination of AST IV and PNS inhibits pyroptosis in rats with cerebral I/R. MCAO induced NLRP3 inflammasome activation in the ischemic area, as evidenced by the increased expression of NLRP3 inflammasome components in the model group compared with the sham group. These included NLRP3, ASC, caspase-1, IL-1 $\beta$ and IL-18 (Fig. 4A-F). The results of the present study also revealed that MCAO significantly increased the expression of GSDMD and GSDMD-N 
A

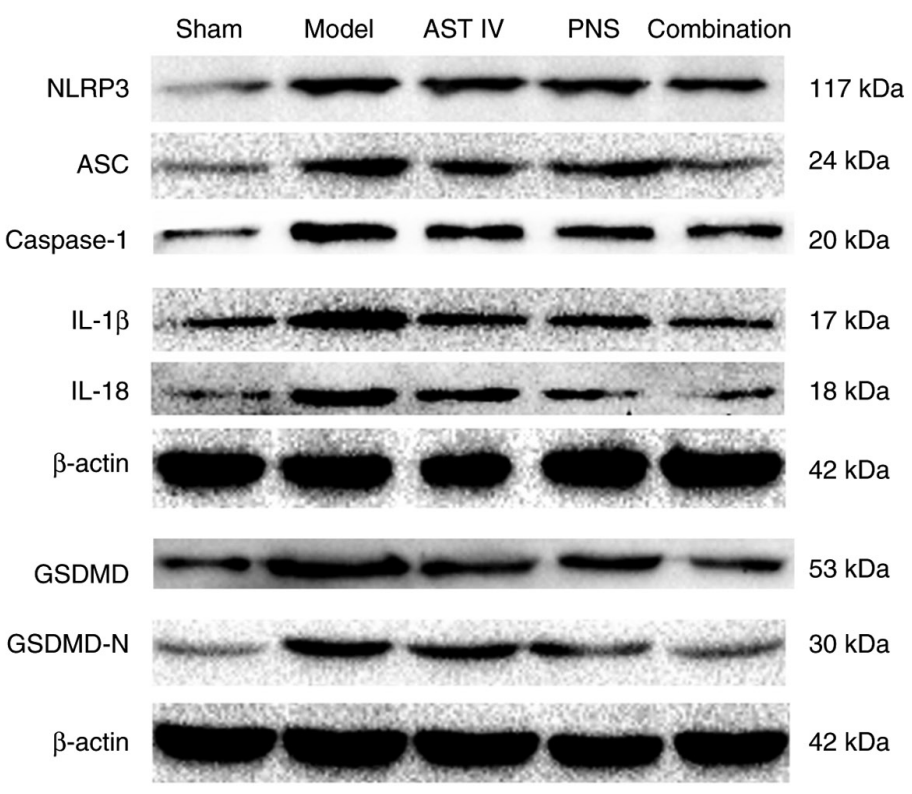

$\mathrm{E}$

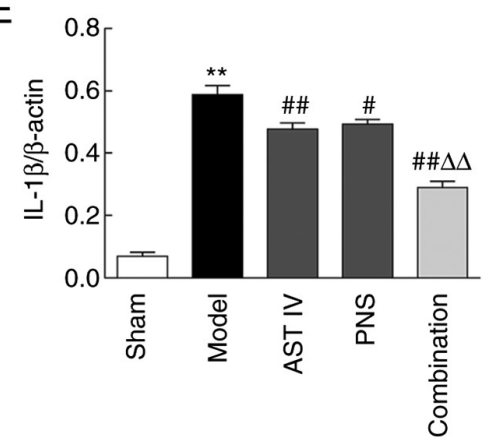

B
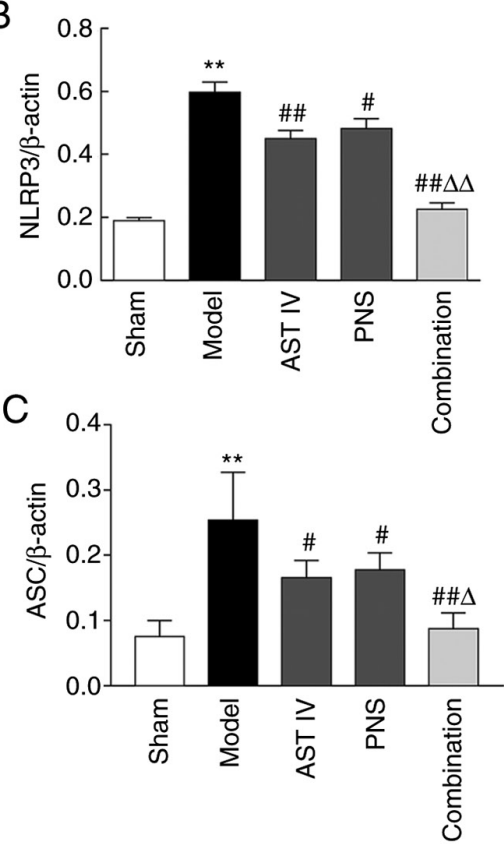

$\mathrm{F}$
G

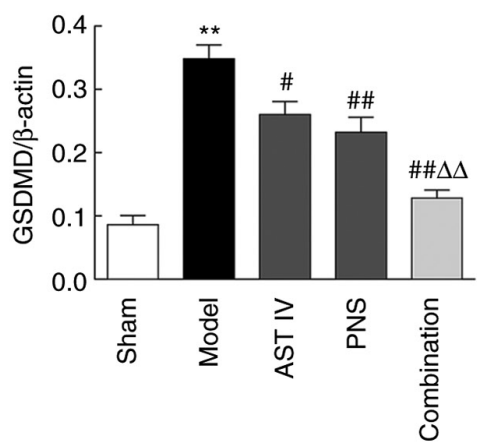

$\mathrm{H}$

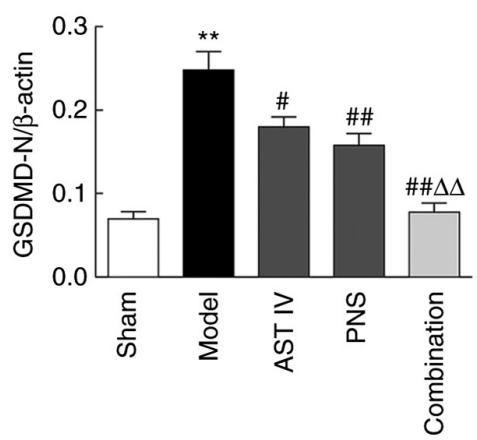

Figure 4. Combination treatment with AST IV and PNS suppresses pyroptosis in rats with cerebral ischemia-reperfusion. (A) Western blot analysis of pyroptosis-associated proteins ( $\mathrm{n}=5$ ). $\beta$-actin was used as the internal reference. Quantification of (B) NLRP3, (C) ASC, (D) caspase-1, (E) IL-1 $\beta$, (F) IL-18, (G) GSDMD and (H) GSDMD-N protein expression levels ( $n=5)$. The groups are as follows: i) Sham, rats with sham operation; ii) model, rats with MCAO; iii) AST IV, rats with MCAO $+28 \mathrm{mg} / \mathrm{kg}$ AST IV; iv) PNS, rats with MCAO + $80 \mathrm{mg} / \mathrm{kg}$ PNS; and v) combination, rats with MCAO + $28 \mathrm{mg} / \mathrm{kg}$ AST IV $+80 \mathrm{mg} / \mathrm{kg}$ PNS. ${ }^{* *} \mathrm{P}<0.01$ vs. Sham; ${ }^{*} \mathrm{P}<0.05,{ }^{\# \#} \mathrm{P}<0.01$ vs. Model; ${ }^{\Delta} \mathrm{P}<0.05,{ }^{\Delta \Delta} \mathrm{P}<0.01$ vs. AST IV or PNS. ASC, apoptosis-associated speck-like protein containing a caspase activation and recruitment domain; AST IV, astragaloside IV; GSDMD, gasdermin D; MCAO, middle cerebral artery occlusion; N, N-terminal; NLRP3, nucleotide-binding oligomerization domain, leucine-rich repeat and pyrin domain containing 3; PNS, Panax notoginseng saponins.

in the cerebral ischemic region compared with the sham group, indicating that $\mathrm{MCAO}$ induced pyroptosis in rats (Fig. 4G and H). Furthermore, the MCAO-induced expression of these proteins was inhibited by treatment with AST IV, PNS or a combination of both, compared with the model group (Fig. 4A-H). The combination of both drugs was more effective than either AST IV or PNS alone in inhibiting such expression (Fig. 4A-H).
Combination of AST IV and PNS reduces the expression of necroptosis-related proteins. RIP1, RIP3 and MLKL are necroptosis-related proteins (8). The results of the present study revealed that the expression of RIP1 and RIP3 and ratio of MLKL and p-MLKL significantly increased in the cerebral cortices of rats in the model group compared with the sham group (Fig. 5A-E). Furthermore, treatment with AST IV and PNS alone or in combination significantly suppressed 
A

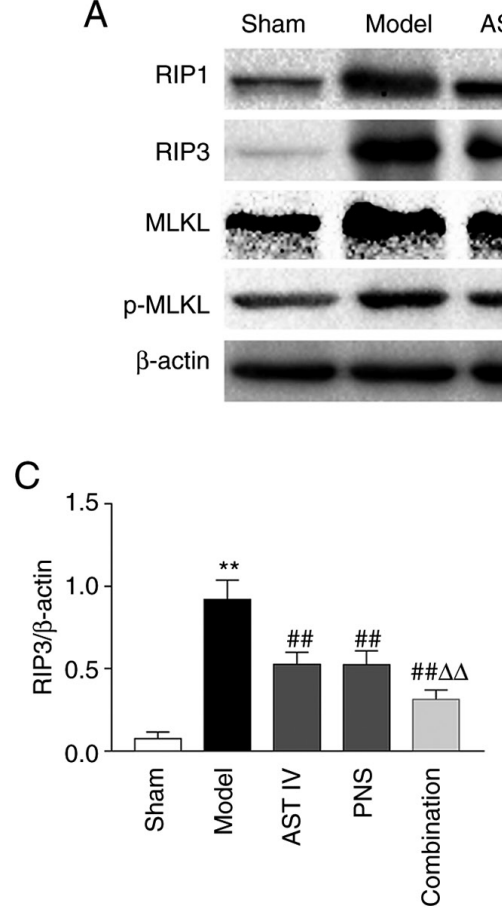

AST IV PNS Combination
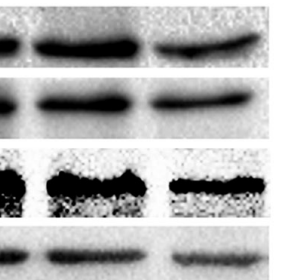

$-1$

$54 \mathrm{kDa}$

$42 \mathrm{kDa}$
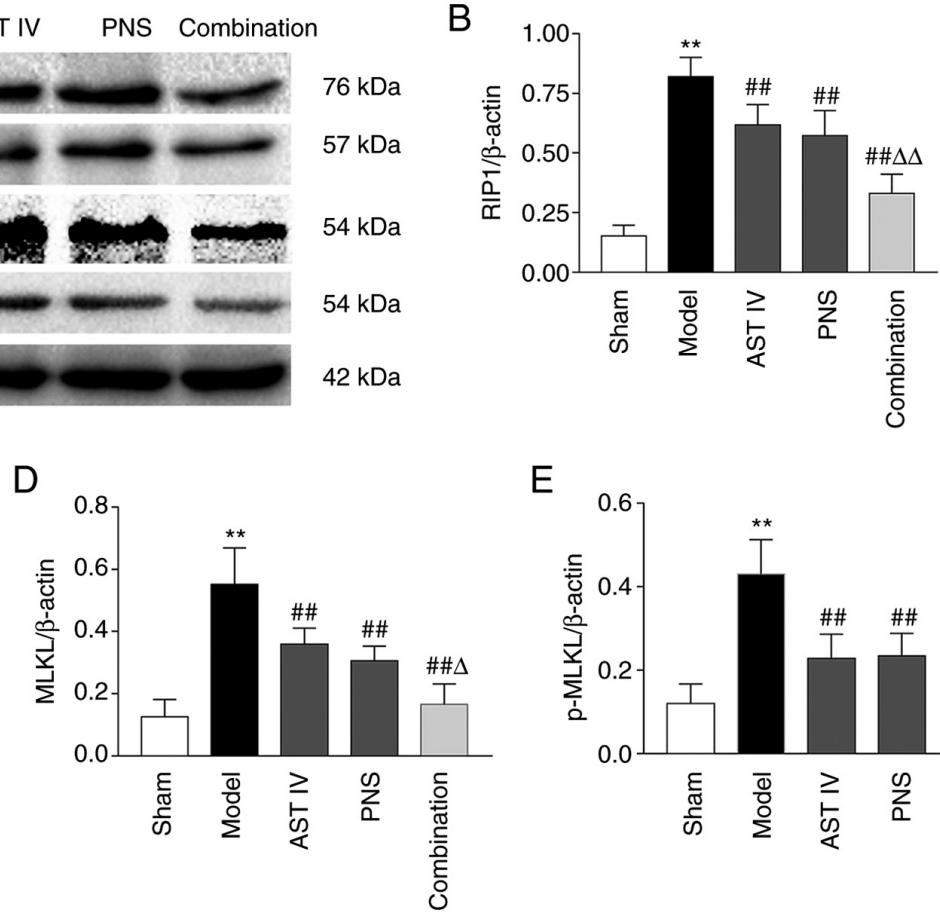

E

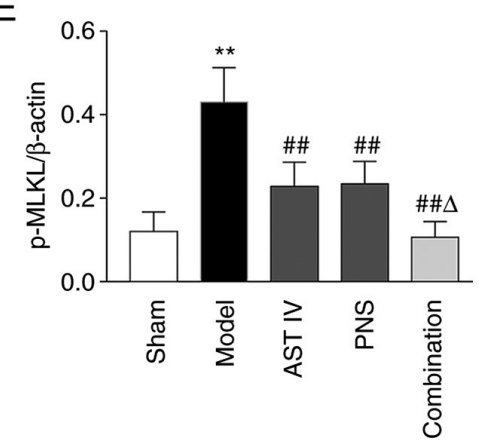

Figure 5. Combination treatment with AST IV and PNS decreases necroptosis-related protein expression. (A) Western blot analysis of necroptosis-related proteins ( $\mathrm{n}=5$ ). $\beta$-actin was used as the internal reference. Quantification of (B) RIP1, (C) RIP3, (D) MLKL and (E) p-MLKL protein expression levels $(\mathrm{n}=5)$. The groups are as follows: i) Sham, rats with sham operation; ii) model, rats with MCAO; iii) AST IV, rats with MCAO + $28 \mathrm{mg} / \mathrm{kg}$ AST IV; iv) PNS, rats with MCAO + $80 \mathrm{mg} / \mathrm{kg}$ PNS; and v) combination, rats with MCAO + $28 \mathrm{mg} / \mathrm{kg}$ AST IV $+80 \mathrm{mg} / \mathrm{kg}$ PNS. ${ }^{* *} \mathrm{P}<0.01 \mathrm{vs}$. Sham; ${ }^{\# / *} \mathrm{P}<0.01 \mathrm{vs}$. Model; ${ }^{\Delta} \mathrm{P}<0.05,{ }^{\Delta \Delta} \mathrm{P}<0.01$ vs. AST IV or PNS. AST IV, astragaloside IV; MCAO, middle cerebral artery occlusion; MLKL, mixed-lineage kinase domain-like protein; p, phosphorylated; PNS, Panax notoginseng saponins; RIP, receptor-interacting protein kinase.

MCAO-induced increases in RIP1, RIP3, MLKL and p-MLKL levels in the rats compared with the model group (Fig. 5A-E). Moreover, the expression of these proteins in the cerebral cortices of rats treated with the drug combination was significantly lower compared with the protein expression levels in rats treated with either AST IV or PNS alone (Fig. 5A-E).

\section{Discussion}

In the present study, an MCAO rat model similar to human cerebral I/R was established, and the effects of AST IV and PNS treatment, alone or in combination, on cerebral $\mathrm{I} / \mathrm{R}$ injury were detected by measuring the cerebral infarct volume, NDS and cerebral pathomorphological indicators. The results revealed that cerebral infarction volume, NDS and the number of damaged cells were significantly increased following cerebral $\mathrm{I} / \mathrm{R}$, and $\mathrm{H} \& \mathrm{E}$ staining indicated that cell injury was markedly increased. Intervention with AST IV, PNS or a combination of both led to a decrease in all of the aforementioned indicators. In addition, the effects of the drug combination were stronger than those of AST IV or PNS alone. Previous studies have demonstrated that both AST IV and PNS have protective effects against I/R injury to the brain, and primary components of A. membranaceus and $P$. notoginseng have been demonstrated to have synergistic effects in the treatment of I/R injury (12-17). The results of the present study were consistent with those of the preceding studies, demonstrating that AST IV and PNS reduced cerebral I/R injury and that the combination of both drugs exhibited the largest effect on cerebral I/R injury in rats.

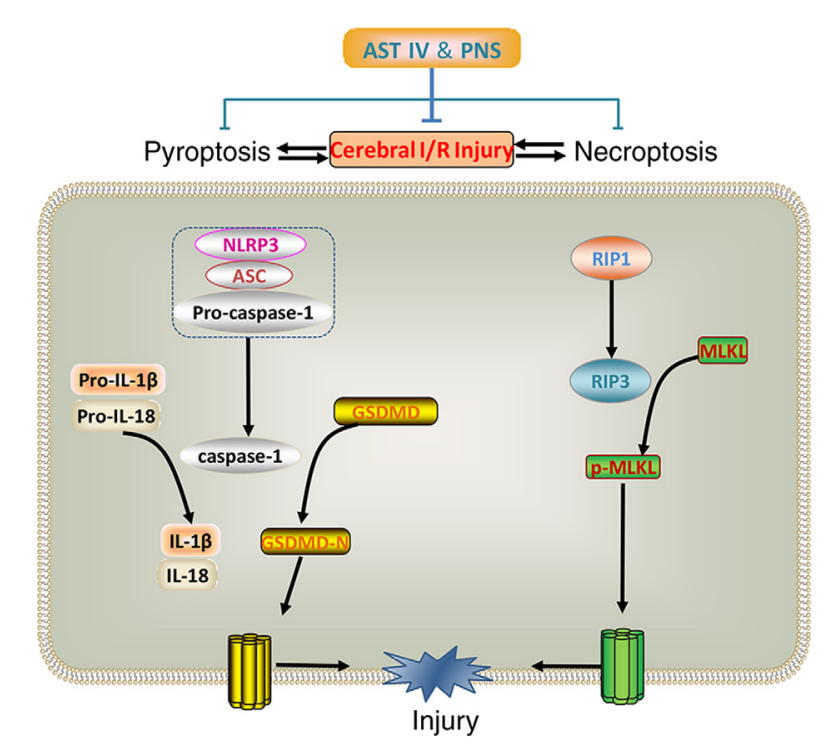

Figure 6. Combination treatment with AST IV and PNS inhibits pyroptosis and necroptosis to attenuate cerebral I/R injury. Pyroptosis and necroptosis are activated in cerebral I/R. Components of the NLRP3 inflammasome, including NLRP3 and ASC, cleave pro-caspase-1 and releases caspase-1.Caspase-1 cleaves pro-IL-1 $\beta / 18$ into inflammatory factors IL-1 $\beta$ and IL-18, and cleaves GSDMD into GSDMD-N, inducing pyroptosis. Through RIP3, RIP1 phosphorylates MLKL into p-MLKL, which induces necroptosis. Both pyroptosis and necroptosis lead to cell death and cerebral I/R injury. The combination of AST IV and PNS suppresses the expression of these proteins and reduces cerebral I/R injury. ASC, apoptosis-associated speck-like protein containing a caspase activation and recruitment domain; AST IV, astragaloside IV; GSDMD, gasdermin D; I/R, ischemia-reperfusion; MLKL, mixed-lineage kinase domain-like protein; N, N-terminal; NLRP3, nucleotide-binding oligomerization domain, leucine-rich repeat and pyrin domain containing 3; p, phosphorylated; PNS, Panax notoginseng saponins; RIP, receptor-interacting protein kinase. 
The expression of GSDMD-N in cells is sufficient to induce pyroptosis and necroptosis, and the NLRP3 inflammasome pathway is the classical pathway for activating cleavage of GSDMD (18). Extracellular stimulation activates NLRP3, which activates caspase-1; caspase-1 in turn cleaves the pro-inflammatory factors IL-1 $\beta$ and IL-18, and simultaneously cleaves GSDMD into GSDMD-N to form the membrane pore (19). Pro-inflammatory factors are released through this membrane pore into the extracellular space, causing inflammation (20). Therefore, the expression levels of NLRP3, ASC, caspase-1, IL-1 $\beta$, IL-18, GSDMD and GSDMD-N are key indicators for verifying NLRP3 inflammasome activation and, consequently, pyroptosis (21). In the present study, the effects of AST IV, PNS or a combination of both on the expression of cerebral cortical NLRP3, ASC, caspase-1, IL-1 $\beta$, IL-18, GSDMD and GSDMD-N were observed in MCAO rats, and the mechanisms underlying AST IV and PNS in I/R injury were investigated.

The results of the present study demonstrated that expression of the aforementioned proteins in the rat cerebral cortex increased significantly following cerebral I/R, which was consistent with the results of a previous study in vitro (21). This indicated that NLRP3 inflammasome-mediated pyroptosis activation occurred in cerebral I/R. In addition, the data of the present study revealed that expression of these proteins in the cerebral cortices of model rats was significantly decreased in the AST IV, PNS and combination groups, which suggested that AST IV and PNS may inhibit NLRP3 inflammasome-mediated activation of pyroptosis in rats with cerebral I/R. It has previously been reported that glycosides serve a neuroprotective role by inhibiting pyroptosis of neurons following brain I/R injury, and that the underlying mechanism is closely associated with NLRP3 inflammasome-related pyroptosis (16). The subsequent results of the current study demonstrated that the combination of AST IV and PNS was more effective than either drug alone in inhibiting pyroptosis. A recent study reported that the inhibition of NLRP3 inflammasome-mediated pyroptosis activation attenuated I/R injury (17). Collectively, these results suggested that AST IV and PNS attenuated cerebral I/R injury by suppressing NLRP3 inflammasome-mediated pyroptosis activation.

The most typical induction of necroptosis is mediated by RIP3 and its substrate MLKL (22). RIP3-mediated MLKL phosphorylation leads to p-MLKL oligomerization, and the oligomers translocate to the plasma membrane to form channels (19), which leads to membrane rupture. Consequently, a number of damage-associated molecular patterns, such as IL- $1 \alpha$, IL-1 $\beta$ and IL-18, are released following necrotic cell rupture, which is a strong trigger for inflammation (22). Therefore, RIP1, RIP3, MLKL and p-MLKL are key factors for evaluating the activation of necroptosis (8).

To further explore the regulatory roles of AST IV and PNS in necroptosis in cerebral $\mathrm{I} / \mathrm{R}$, the effects of both on cortical RIP1, RIP3 expression, and p-MLKL/MLKL ratios in cerebral I/R were observed. The expression of all four proteins in the cerebral cortices of rats increased significantly following cerebral I/R, suggesting that cerebral I/R induced programmed necroptosis, which was consistent with a recent study (8). These events were significantly inhibited after treatment with AST IV, PNS or a combination of both, which indicated that both drugs suppressed necroptosis induced by cerebral I/R in rats. The results of the present study further revealed that a combination of AST IV and PNS inhibited necroptosis in the cerebral cortices of rats more potently compared with treatment with either drug alone. Although recent studies have demonstrated that inhibition of necroptosis improved cerebral I/R injury $(8,23)$, the results of the present study are the first to indicate that the underlying mechanisms by which AST IV and PNS reduce I/R injury are associated with necroptotic inhibition.

In conclusion, AST IV, PNS or a combination of both exerted protective effects in brain I/R injury. The underlying mechanisms were associated with inhibition of NLRP3 inflammasome-mediated pyroptosis and RIP1-, RIP3- and MLKL-mediated necroptosis in cerebral I/R (Fig. 6).

\section{Acknowledgements}

Not applicable.

\section{Funding}

This work was supported by The National Natural Science Foundation of China (grant no. 81503385), The Scientific Research Fund of the Hunan Provincial Education Department (grant no. 19B436), The Hunan Administration of Traditional Chinese Medicine Science Foundation (grant no. 2021215), The Scientific Research Foundation of Hunan University of Chinese Medicine (grant no. 202024) and The Excellent Teaching Team of Postgraduate in Hunan Province [Teaching Team of Postgraduate in Basic Medicine; grant no. (2019)370-118].

\section{Availability of data and materials}

The datasets used and/or analyzed during the current study are available from the corresponding author on reasonable request.

\section{Authors' contributions}

BT and CQD conceived the study and designed the experiments. BT performed the experiments. BT and XS analyzed the data and drafted the manuscript. BT and CQD reviewed and revised the manuscript. BT and CQD confirm the authenticity of all the raw data All authors have read and approved the final manuscript.

\section{Ethics approval and consent to participate}

This study was approved by the Animal Ethics Committee of Hunan University of Chinese Medicine (Changsha, China; approval no. 43004700006817).

\section{Patient consent for publication}

Not applicable.

\section{Competing interests}

The authors declare that they have no competing interests. 


\section{References}

1. Wu MY, Yiang GT, Liao WT, Tsai AP, Cheng YL, Cheng PW, Li CY and Li CJ: Current mechanistic concepts in ischemia and reperfusion injury. Cell Physiol Biochem 46: 1650-1667, 2018.

2. Shan B, Pan H, Najafov A and Yuan J: Necroptosis in development and diseases. Genes Dev 32: 327-340, 2018.

3. Barbosa LA, Fiuza PP, Borges LJ, Rolim FA, Andrade MB Luz NF, Quintela-Carvalho G, Lima JB, Almeida RP, Chan FK, et al: RIPK1-RIPK3-MLKL-Associated Necroptosis Drives Leishmania infantum Killing in Neutrophils. Front Immunol 9: 1818, 2018.

4. Shi J, Gao W and Shao F: Pyroptosis: Gasdermin-mediated programmed necrotic cell death. Trends Biochem Sci 42 : 245-254, 2017.

5. Lin SY, Hsieh SY, Fan YT, Wei WC, Hsiao PW, Tsai DH, Wu TS and Yang NS: Necroptosis promotes autophagy-dependent upregulation of DAMP and results in immunosurveillance. Autophagy 14: 778-795, 2018.

6. Zhang D, Qian J, Zhang P, Li H, Shen H, Li X and Chen G: Gasdermin D serves as a key executioner of pyroptosis in experimental cerebral ischemia and reperfusion model both in vivo and in vitro. J Neurosci Res 97: 645-660, 2019.

7. An P, Xie J, Qiu S, Liu Y, Wang J, Xiu X, Li L and Tang M Hispidulin exhibits neuroprotective activities against cerebral ischemia reperfusion injury through suppressing NLRP3-mediated pyroptosis. Life Sci 232: 116599, 2019.

8. Li X, Cheng S, Hu H, Zhang X, Xu J, Wang R and Zhang P: Progranulin protects against cerebral ischemia-reperfusion (I/R) injury by inhibiting necroptosis and oxidative stress. Biochem Biophys Res Commun 521: 569-576, 2020.

9. Huang XP, Ding H, Yang XQ, Li JX, Tang B, Liu XD, Tang YH and Deng CQ: Synergism and mechanism of Astragaloside IV combined with Ginsenoside $\operatorname{Rg} 1$ against autophagic injury of PC12 cells induced by oxygen glucose deprivation/reoxygenation. Biomed Pharmacother 89: 124-134, 2017.

10. Huang XP, Ding H, Wang B, Qiu YY, Tang YH, Zeng R and Deng CQ: Effects of the main active components combinations of Astragalus and Panax notoginseng on energy metabolism in brain tissues after cerebral ischemia-reperfusion in mice. Pharmacogn Mag 11: 732-739, 2015.

11. Huang XP, Qiu YY, Wang B, Ding H, Tang YH, Zeng R and Deng CQ: Effects of Astragaloside IV combined with the active components of Panax notoginseng on oxidative stress injury and nuclear factor-erythroid 2-related factor 2/heme oxygenase-1 signaling pathway after cerebral ischemia-reperfusion in mice. Pharmacogn Mag 10: 402-409, 2014.

12. Huang XP, Ding H, Lu JD, Tang YH, Deng BX and Deng CQ Effects of the combination of the main active components of astragalus and panax notoginseng on inflammation and apoptosis of nerve cell after cerebral ischemia-reperfusion. Am J Chin Med 43: 1419-1438, 2015
13. Longa EZ, Weinstein PR, Carlson S and Cummins R: Reversible middle cerebral artery occlusion without craniectomy in rats. Stroke 20: 84-91, 1989.

14. Zhang X, Yan H, Yuan Y, Gao J, Shen Z, Cheng Y, Shen Y, Wang RR, Wang X, Hu WW, et al: Cerebral ischemia-reperfusion-induced autophagy protects against neuronal injury by mitochondrial clearance. Autophagy 9: 1321-1333, 2013.

15. Wang HL, Zhou QH, Xu MB, Zhou XL and Zheng GQ: Astragaloside IV for experimental focal cerebral ischemia: Preclinical evidence and possible mechanisms. Oxid Med Cell Longev 2017: 8424326, 2017.

16. She Y, Shao L, Zhang Y, Hao Y, Cai Y, Cheng Z, Deng C and Liu X: Neuroprotective effect of glycosides in Buyang Huanwu Decoction on pyroptosis following cerebral ischemia-reperfusion injury in rats. J Ethnopharmacol 242: 112051, 2019.

17. Liu D, Dong Z, Xiang F, Liu H, Wang Y, Wang Q and Rao J: Dendrobium alkaloids promote neural function after cerebral ischemia-reperfusion injury through inhibiting pyroptosis induced neuronal death in both in vivo and in vitro models. Neurochem Res 45: 437-454, 2020.

18. Shi J, Zhao Y, Wang K, Shi X, Wang Y, Huang H, Zhuang Y, Cai T, Wang F and Shao F: Cleavage of GSDMD by inflammatory caspases determines pyroptotic cell death. Nature 526: $660-665,2015$

19. Kolbrink B, Riebeling T, Kunzendorf U and Krautwald S: Plasma membrane pores drive inflammatory cell death. Front Cell Dev Biol 8: 817, 2020.

20. Heilig R, Dick MS, Sborgi L, Meunier E, Hiller S and Broz P: The Gasdermin-D pore acts as a conduit for IL-1 $\beta$ secretion in mice. Eur J Immunol 48: 584-592, 2018.

21. DiaoMY,Zhu Y,Yang J,Xi SS, Wen X,GuQ and Hu W: Hypothermia protects neurons against ischemia/reperfusion-induced pyroptosis via m6A-mediated activation of PTEN and the PI3K/Akt/GSK-3 $\beta$ signaling pathway. Brain Res Bull 159: 25-31, 2020.

22. Pasparakis $M$ and Vandenabeele P: Necroptosis and its role in inflammation. Nature 517: 311-320, 2015.

23. Naito MG, Xu D, Amin P, Lee J, Wang H, Li W, Kelliher M, Pasparakis M and Yuan J: Sequential activation of necroptosis and apoptosis cooperates to mediate vascular and neural pathology in stroke. Proc Natl Acad Sci USA 117: 4959-4970, 2020. International (CC BY-NC-ND 4.0) License. 